\title{
Integral points and effective cones of moduli spaces of stable maps
}

\author{
Brendan Hassett ${ }^{*}$ and Yuri Tschinkel ${ }^{\dagger}$ \\ May 8, 2002
}

\begin{abstract}
Consider the Fulton-MacPherson configuration space of $n$ points on $\mathbb{P}^{1}$, which is isomorphic to a certain moduli space of stable maps to $\mathbb{P}^{1}$. We compute the cone of effective $\mathfrak{S}_{n}$-invariant divisors on this space. This yields a geometric interpretation of known asymptotic formulas for the number of integral points of bounded height on compactifications of $\mathrm{SL}_{2}$ in the space of binary forms of degree $n \geq 3$.
\end{abstract}

\section{Contents}

\begin{tabular}{lll}
\hline 0 & Introduction & 2 \\
\hline
\end{tabular}

\begin{tabular}{|ll}
\hline & Generalities
\end{tabular}

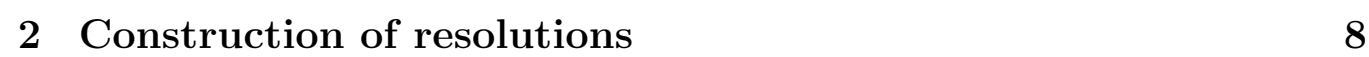

$\begin{array}{lll}3 & \text { Verification of exponents } & 15\end{array}$

\begin{tabular}{|lr}
4 The invariant effective cone & 18
\end{tabular}

$\begin{array}{lll}5 & \text { Final remarks } & 23\end{array}$

*Partially supported by NSF grant 0196187

${ }^{\dagger}$ Partially supported by NSF grant 0100277 and the Clay Foundation 


\section{Introduction}

In this paper, we compute the $\mathfrak{S}_{n}$-invariant cone of effective divisors of the Fulton-MacPherson configuration space of $n$ points on $\mathbb{P}^{1}$. This space is isomorphic to the moduli space $\overline{\mathcal{M}}_{0, n}\left(\mathbb{P}^{1}, 1\right)$ of stable maps of degree one from genus zero curves with $n$ marked points to $\mathbb{P}^{1}$. We also compute the effective cone of the generic fiber of the natural map

$$
\overline{\mathcal{M}}_{0, n}\left(\mathbb{P}^{1}, 1\right) / \mathfrak{S}_{n} \rightarrow \overline{\mathcal{M}}_{0, n} / \mathfrak{S}_{n}
$$

Our motivation is to provide a geometric explanation of a formula, obtained by Duke, Rudnick and Sarnak, giving the asymptotic behavior of the number of binary forms of degree $n$ with fixed discriminant and bounded integral coefficients. This fits into a larger program to predict and prove asymptotic formulas for the number of rational and integral points of bounded height on algebraic varieties.

We introduce a counting function for integral points on an algebraic variety as follows: given a variety $U$ over a ring of integers $\mathfrak{o}$ and functions $g_{1}, \ldots, g_{n}$, regular on $U$, define

$$
N(U, B):=\left\{x \in U(\mathfrak{o}) \mid \max _{j}\left(\left\|g_{j}(x)\right\|\right) \leq B\right\}
$$

where $\|\cdot\|$ is a valuation on $\mathfrak{o}$. This is finite only when the functions $g_{j}$ give an embedding of $U$.

It is most natural to interpret the functions $g_{j}$ as sections of a line bundle $L$ on a projective compactification $X \supset U$ defined over the fraction field $F$ of $\mathfrak{o}$. The fact that the sections embed $U$ implies that $L$ is big, i.e., is contained in the interior of the effective cone $\Lambda_{\text {eff }}(X)$ of $X$. Therefore, in order to describe all natural counting functions on open subsets of $X$ we need to compute its effective cone. Furthermore, in many cases it can be proved that the asymptotic properties of $N(U, B)$ are intimately related to the structure of this cone.

Let $P(\mathbf{x})=P\left(x_{0}, \ldots, x_{r}\right)$ be a homogeneous polynomial of degree $n$ in $r+1$ variables. A standard heuristic in number theory predicts that the number

$$
N_{P}(B):=\left\{\mathbf{x} \mid \max \left(\left|x_{j}\right|\right) \leq B, P(\mathbf{x})=0, \text { and } \mathbf{x} \in \mathbb{Z}^{r+1}\right\}
$$


of integral solutions of the equation $P(\mathbf{x})=0$ of "height" $\leq B$ grows asymptotically like $B^{r+1-n}$ as $B \rightarrow \infty$. When the number of variables is $\gg 2^{n}$, the affine variety $V_{P}$ defined by $P=0$ is smooth and there are no local obstructions, an asymptotic formula can be established using the classical circle method in analytic number theory (see [3, 25] and the references therein). Of course, there may be difficulties when the number of variables is small or the variety $V_{P}$ is singular.

The following example appeared in the paper by Duke, Rudnick and Sarnak [6]. Consider the vector space of binary forms of degree $n$

$$
x_{n} z^{n}+x_{n-1} z^{n-1} w+\ldots+x_{0} w^{n} .
$$

The algebraic group $\mathrm{SL}_{2}$ acts on this space by coordinate substitutions. When $n=3$, the discriminant form

$$
\operatorname{disc}\left(x_{0}, \ldots, x_{3}\right):=27 x_{0}^{2} x_{3}^{2}-18 x_{0} x_{1} x_{2} x_{3}+4 x_{0} x_{2}^{3}+4 x_{1}^{3} x_{3}-x_{1}^{2} x_{2}^{2}
$$

generates the ring of $\mathrm{SL}_{2}$-invariants. Then there exists a constant $c>0$ so that

$$
N_{\text {disc }-1}(B)=c B^{2 / 3}(1+o(1))
$$

as $B \rightarrow \infty$. Note that the exponent $2 / 3$ is larger than what is predicted by the standard heuristic.

More generally, one has the

Theorem 0.1 [6] Fix a generic binary form $f$ of degree $n \geq 3$ with integral coefficients. Let $N(B)$ be the number of binary forms $\mathrm{SL}_{2}(\mathbb{Z})$-equivalent to $f$ with coefficients bounded by $B$. Then there exists a $c>0$ such that

$$
N(B)=c B^{2 / n}(1+o(1)),
$$

as $B \rightarrow \infty$.

We give a geometric interpretation of the exponent $2 / n$ in Theorem 0.1 . To this end, we refine the heuristics for counting integral points to take into account singularities of the relevant varieties (see Conjecture 1.6). We verify that Conjecture 1.6 is consistent with Theorem 0.1] in Theorem 2.1] Its proof involves the computations of effective cones alluded to above.

Acknowledgments: We are grateful to the Alfréd Rényi Institute of the Hungarian Academy of Sciences for organizing the conference at which much of this work was done. 


\section{Generalities}

\subsection{Singularities of pairs and effective cones}

We work over a field of characteristic zero. Let $X$ be a normal projective variety with canonical class $K_{X}$ and let $D$ be a reduced effective Weil divisor of $X$.

Definition 1.1 A good pair $(X, D)$ consists of a smooth projective variety $X$ and a strict normal crossings divisor $D$ in $X$. This means that all irreducible components of $D$ are smooth and intersect transversally.

Let $(X, D)$ be a good pair and let $\Lambda_{\text {eff }}(X)$ denote the closed cone of effective divisors classes of $X$; a divisor is big exactly when its class is in the interior of this cone. Define

$$
a(L, D):=\inf \left\{a \in \mathbb{R} \mid a L+\left(K_{X}+D\right) \in \Lambda_{\text {eff }}(X)\right\},
$$

where we identify line bundles and their divisor classes. Note that $a(L, D)$ is a positive real number whenever $-\left(K_{X}+D\right)$ is big. The constant $-a(L, D)$ is called the log-Kodaira energy of $L$ (see [10]).

If $(X, D)$ is not good then resolution of singularities implies the existence of a good resolution $\rho:(\tilde{X}, \tilde{D}) \rightarrow(X, D)$. Precisely, $(\tilde{X}, \tilde{D})$ is a good pair, $\rho$ a birational projective morphism, and $\tilde{D}$ is the union of the exceptional divisors of $\rho$ and the proper transform of $D$. Recall that $(X, D)$ is logcanonical if $K_{X}+D$ is $\mathbb{Q}$-Cartier and

$$
K_{\tilde{X}}+\tilde{D} \equiv_{\mathbb{Q}} \rho^{*}\left(K_{X}+D\right)+\sum d_{j} E_{j},
$$

where the $E_{j}$ are the exceptional divisors of $\rho$ and $d_{j} \geq 0$ for all $j$.

Example 1.2 When $X$ is a smooth surface, $(X, D)$ is log-canonical only when the curve $D$ is smooth or nodal. If $X$ is smooth of arbitrary dimension, $D$ must have at worse nodes in codimension one.

If $L$ is a line bundle on $X$ put

$$
a(L, D):=a\left(\rho^{*} L, D^{t}\right),
$$

where $D^{t} \subset \tilde{X}$ is the total transform of $D$. Note that $a(L, D)$ is computed on $\tilde{X}$. 
Proposition 1.3 Let $(X, D)$ be a log-canonical pair and assume that $X-D$ has canonical singularities. If $L$ is a big line bundle on $X$ then

$$
a(L, D)=\inf \left\{a \in \mathbb{R} \mid a L+\left(K_{X}+D\right) \in \Lambda_{\text {eff }}(X)\right\} .
$$

In particular, a $(L, D)$ does not depend on the choice of a desingularization.

Proof. Choose a good resolution $\rho:(\tilde{X}, \tilde{D}) \rightarrow(X, D)$, so that

$$
K_{\tilde{X}}+\tilde{D}-\sum d_{j} E_{j}=\rho^{*}\left(K_{X}+D\right)
$$

where $d_{j} \geq 0$, and $d_{j} \geq 1$ if $\rho\left(E_{j}\right) \not \subset D$. In particular, each exceptional divisor not contained in the total transform $D^{t}$ has $\log$ discrepancy $\geq 1$. Therefore, we have

$$
\alpha \rho^{*}(L)+K_{\tilde{X}}+D^{t}-\sum d_{j}^{\prime} E_{j}=\rho^{*}\left(K_{X}+D+\alpha L\right),
$$

with each $d_{j}^{\prime} \geq 0$. For any $\mathbb{Q}$-Cartier divisor $M$ on $X$ and effective divisor $\sum d_{j}^{\prime} E_{j}$ supported in the exceptional locus of $\rho, M$ is effective iff $\rho^{*}(M)+\sum d_{j}^{\prime} E_{j}$ is effective.

Proposition 1.4 Let $\left(X_{1}, D_{1}\right)$ and $\left(X_{2}, D_{2}\right)$ be log-canonical pairs, so that $X_{1}-D_{1}$ and $X_{2}-D_{2}$ have canonical singularities. Assume that $\pi: X_{1} \rightarrow X_{2}$ is a finite dominant morphism so that

$$
\pi^{*}\left(K_{X_{2}}+D_{2}\right)=K_{X_{1}}+D_{1}
$$

Let $L$ be a big divisor on $X_{2}$. Then $a\left(L, D_{2}\right)=a\left(\pi^{*}(L), D_{1}\right)$.

In fact, it suffices to assume that either $\left(X_{1}, D_{1}\right)$ or $\left(X_{2}, D_{2}\right)$ satisfies the singularity condition [17, Sect. 20.3].

Proof. Given a finite dominant morphism $\pi: X_{1} \rightarrow X_{2}$ and a $\mathbb{Q}$-Cartier divisor $M$ on $X_{2}, M$ is effective iff $\pi^{*}(M)$ is effective. Indeed, the divisor $\pi_{*} \pi^{*} M$ is defined and equal to $\operatorname{deg}(\pi) M$. Combining this with Proposition 1.3 gives the result.

Remark 1.5 Let $(X, D)$ be a log terminal pair so that $X-D$ has singularities which are not canonical. Then our definition of the Kodaira energy 
differs slightly from Fujita's [10]. In applications to integral points, we are interested in invariants of the open variety $X-D$. In Fujita's definition, on passing from $(X, D)$ to a good resolution, any exceptional divisors over $X-D$ with negative discrepancy must be added to the boundary. This changes the open variety.

\section{$1.2 \quad$ Integral points}

Retain the notation from the previous section and assume that $X$ and $D$ are defined over a number field $F$. Let $\mathfrak{o}_{S}$ denote the ring of integers of $F$, where $S$ is a finite set of nonarchimedean places of $F$. Fix models $\mathcal{X}$ and $\mathcal{D}$ flat and proper over the ring of integers $\mathfrak{o}_{S}$. A $(D, S)$-integral point is an $\mathfrak{o}_{S}$-point of $(\mathcal{X}-\mathcal{D})$. In particular, if $D=\emptyset$ an integral point is the same as a rational point on $X$.

Let $\mathcal{L}$ be a very ample metrized line bundle on $X, U \subset X$ a Zariski open subset and $\mathcal{U}$ a model of $U$ over $\mathfrak{o}_{S}$. Let $S$ be a finite set of places in $F$, including the archimedean places. Let

$$
N(\mathcal{U}, \mathcal{L}, B):=\#\left\{x \in \mathcal{U}\left(\mathfrak{o}_{S}\right) \mid H_{\mathcal{L}}(x) \leq B\right\}
$$

denote the number of $(D, S)$ integral points on $U$ of $\mathcal{L}$-height bounded by $B$. A natural extrapolation of Vojta's conjecture about integral and rational points on varieties of (log-)general type [27] and Batyrev-Manin conjectures about rational points of bounded height on Fano varieties 9, 1] would be:

Conjecture 1.6 For any $\epsilon>0$, there exists a dense Zariski open subset $U \subset X$ such that

$$
N(\mathcal{U}, \mathcal{L}, B) \ll B^{a(L, D)+\epsilon}
$$

as $B \rightarrow \infty$. If $-\left(K_{X}+D\right)$ is big then

$$
N(\mathcal{U}, \mathcal{L}, B) \gg B^{a(L, D)-\epsilon},
$$

as $B \rightarrow \infty$, at least after a suitable finite extension of $F$ and $S$.

The statement is independent of the choice of $S$ and the choice of a metrization on $L$.

Many precise results about asymptotics of rational and integral points are currently available (see, for example, [9, 2, 4, 21, 22, 6, 7, 8] and the references therein). As far as we know, Conjecture [1.6 is compatible with all of 
them. However, to actually check this compatibility one has to compute the geometric invariants of (some resolution of) the pair $(X, D)$. In particular, one has to determine the effective cone. This can be a formidable task even for rational varieties, e.g., like the moduli space of pointed rational curves $\overline{\mathcal{M}}_{0, n}$ (see [14).

\subsection{Computing effective cones}

Let $X$ be a nonsingular projective variety, perhaps with an action by a finite group $G$. We review strategies for computing the $G$-invariant effective cone $\Lambda_{\mathrm{eff}}(X)^{G}$ and thus the effective cone of the quotient $X / G$ (cf. [16]).

A curve class $[C] \in N_{1}(X)$ is said to be nef if $[C] . D \geq 0$ for each $D \in \Lambda_{\text {eff }}(X)$. A family of curves passing through the generic point of $X$ is automatically nef. Indeed, consider a family $\mathcal{C} \rightarrow B$ of integral projective curves in $X$ and an irreducible codimension-one subvariety $D \subset X$. If, for generic $b \in B$, the fiber $C_{b} \not \subset D$, we have $\left[C_{b}\right] . D \geq 0$.

Fix a collection of effective divisors

$$
\Gamma=\left\{A_{1}, \ldots, A_{m}\right\}
$$

which we expect to generate $\Lambda_{\text {eff }}(X)^{G}$. To prove that $\Gamma$ generates the $(G$ invariant) effective cone, it suffices to find a collection of nef ( $G$-invariant) curve classes

$$
\Xi=\left\{C_{1}, \ldots, C_{\ell}\right\}
$$

so that the cone generated by $\Gamma$ contains the dual to the cone generated by $\Xi$.

In section 4, we shall use a refinement of this method (see [5], 24]). A divisor $D \in \Lambda_{\text {eff }}(X)$ is moving relative to $\Gamma$ if some multiple of $D$ contains no element of $\Gamma$ as a fixed component. Every effective divisor is a sum

$$
M+\sum_{i=1}^{m} A_{i} d_{i}, \quad d_{i} \geq 0
$$

where $M$ is moving relative to $\Gamma$. To prove that $\Gamma$ generates the effective cone, it suffices to show that $M$ is an effective sum of the $A_{i}$.

A curve class is nef relative to $\Gamma$ if $[C] . M \geq 0$ for each $M$ which is moving relative to $\Gamma$. Any family of curves passing through the generic point of some 
$A_{i}$ is nef relative to $\Gamma$. Consequently, to show that $\Gamma$ generates the effective cone, it suffices to find a collection $\Xi$ of curve classes, nef relative to $\Gamma$, so that the cone generated by $\Gamma$ contains the dual to the cone generated by $\Xi$.

\section{Construction of resolutions}

\subsection{Binary forms and $\mathrm{SL}_{2}$-orbit closures}

Let $V$ be a two-dimensional vector space with coordinates $z$ and $w$, equipped with the standard $\mathrm{SL}_{2}$-action. Let $\mathrm{Sym}^{n} V^{*}$ be the space of binary forms of degree $n$

$$
f=x_{0} z^{n}+x_{1} z^{n-1} w+\ldots+x_{n} w^{n} .
$$

It carries an induced action of $\mathrm{SL}_{2}$ by substitution.

Associating to each form $f \neq 0$ its roots $\alpha_{1}, \ldots, \alpha_{n}$ yields a map

$$
\left(\operatorname{Sym}^{n} V^{*}-0\right) \rightarrow \mathbb{P}(V)^{n} / \mathfrak{S}_{n}
$$

and an identification $\mathbb{P}\left(\operatorname{Sym}^{n} V^{*}\right) \simeq \mathbb{P}(V)^{n} / \mathfrak{S}_{n}$. The discriminant of a polynomial $f$ is a homogeneous form in its coefficients $x_{0}, \ldots, x_{n}$ and defines a divisor $D \subset X=\mathbb{P}\left(\operatorname{Sym}^{n} V^{*}\right)$.

Now we may state our main result:

Theorem 2.1 (Computation of Kodaira Energy) Let $f$ be a generic bilinear form of degree $n, X_{f} \subset \mathbb{P}\left(\mathrm{Sym}^{n} V^{*}\right)$ the closure of the $\mathrm{SL}_{2}$-orbit through $f, D_{f}$ the intersection of the discriminant with $X_{f}$, and $L$ the restriction of the standard polarization to $X_{f}$. Then we have

$$
a\left(L, D_{f}\right)=2 / n \text {. }
$$

In particular, Conjecture 1.6 is consistent with Theorem 0.1

To prove this, we require a resolution (i.e., a partial desingularization) of $\left(X_{f}, D_{f}\right)$ on which we may evaluate $a\left(L, D_{f}\right)$ using Proposition 1.3. This resolution will be induced by a natural resolution of $(X, D)$.

Remark 2.2 Example 1.2 shows that $(X, D)$ is far from being log-canonical. When $n=3$, the discriminant has cusps in codimension one: a transverse slice

$$
z^{3}+b z w^{2}+c w^{3}
$$


intersects the discriminant in the cuspidal curve

$$
4 b^{3}+27 c^{2}=0 .
$$

Our resolution of $(X, D)$ will be a $\mathfrak{S}_{n}$-quotient of a natural desingularization for $\left(\mathbb{P}(V)^{n}, \Delta\right)$, where $\Delta$ is the diagonal, i.e., the points lying over the discriminant. Both admit interpretations as moduli spaces of stable maps.

\subsection{Moduli spaces}

Fix an integer $n \geq 3$. Let $\overline{\mathcal{M}}_{0, n}$ denote the Knudsen-Mumford moduli space of stable curves of genus zero with $n$ marked points [18]

$$
\left(C, p_{1}, \ldots, p_{n}\right) \text {. }
$$

Let $\overline{\mathcal{M}}_{0, n}\left(\mathbb{P}^{1}, 1\right)$ denote the Kontsevich moduli space of stable maps of degree one from genus-zero curves with $n$ marked points to $\mathbb{P}^{1}$ [19, 20, 12,

$$
\left(C, p_{1}, \ldots, p_{n}, \mu: C \rightarrow \mathbb{P}^{1}\right) .
$$

This is naturally isomorphic to the Fulton-MacPherson [1] configuration space $\mathbb{P}^{1}[n]$ for $n$ points in $\mathbb{P}^{1}$ (see [12] $\S 0$ ). However, for our purposes it is convenient to use the moduli space notation.

We have the following natural maps:

1. the evaluation map

$$
\begin{aligned}
\overline{\mathcal{M}}_{0, n}\left(\mathbb{P}^{1}, 1\right) & \longrightarrow\left(\mathbb{P}^{1}\right)^{n} ; \\
\left(C, p_{1}, \ldots, p_{n}, \mu\right) & \mapsto\left(\mu\left(p_{1}\right), \ldots \mu\left(p_{n}\right)\right) ;
\end{aligned}
$$

2. forgetting the point $p_{j}$

$$
\begin{aligned}
\phi_{j}: \overline{\mathcal{M}}_{0, n} & \longrightarrow \overline{\mathcal{M}}_{0, n-1}, \quad(n \geq 4) \\
\left(C, p_{1}, \ldots, p_{n}\right) & \mapsto\left(C^{\prime}, p_{1}, \ldots, \hat{p}_{j}, \ldots, p_{n}\right) ; \\
\phi_{j}: \overline{\mathcal{M}}_{0, n}\left(\mathbb{P}^{1}, 1\right) & \longrightarrow \overline{\mathcal{M}}_{0, n-1}\left(\mathbb{P}^{1}, 1\right) \\
\left(C, p_{1}, \ldots, p_{n}, \mu\right) & \mapsto\left(C^{\prime}, p_{1}, \ldots, \hat{p}_{j}, \ldots, p_{n}, \mu^{\prime}\right) .
\end{aligned}
$$

3. taking projective equivalence classes

$$
\begin{aligned}
\psi: \overline{\mathcal{M}}_{0, n}\left(\mathbb{P}^{1}, 1\right) & \longrightarrow \overline{\mathcal{M}}_{0, n} \\
\left(C, p_{1}, \ldots, p_{n}, \mu\right) & \mapsto\left(C^{\prime}, p_{1}, \ldots, p_{n}\right) .
\end{aligned}
$$


$C^{\prime}$ is obtained from $C$ by 'collapsing' the irreducible components which are destabilized when $p_{j}$ (resp., the polarization) is removed.

Finally, we enumerate the boundary divisors of these moduli spaces. For each partition

$$
\{1, \ldots, n\}=S \cup S^{\prime}, \quad 2 \leq|S| \leq\left|S^{\prime}\right| \leq n-2,
$$

consider stable curves

$$
C=\left(\mathbb{P}^{1}, p_{j}, j \in S\right) \cup\left(\mathbb{P}^{1}, p_{j}, j \in S^{\prime}\right),
$$

which form a divisor $\delta_{S, S^{\prime}} \subset \overline{\mathcal{M}}_{0, n}$. The union of these is denoted $\delta$. Note that the $\mathfrak{S}_{n}$-orbits of $\left\{\delta_{S, S^{\prime}}\right\}$ correspond to the integers

$$
|S|=2, \ldots,\lfloor n / 2\rfloor .
$$

For each subset

$$
S \subset\{1, \ldots, n\}, \quad 2 \leq S
$$

consider stable maps

$$
\mu: C=\left(\mathbb{P}^{1}, p_{j}, j \in S\right) \cup\left(\mathbb{P}^{1}, p_{j}, j \in S^{\prime}\right) \longrightarrow \mathbb{P}^{1}
$$

collapsing the first component and mapping the second isomorphically onto $\mathbb{P}^{1}$. These form a divisor $B_{S} \subset \overline{\mathcal{M}}_{0, n}\left(\mathbb{P}^{1}, 1\right)$. The $\mathfrak{S}_{n}$-orbits of $\left\{B_{S}\right\}$ correspond to integers

$$
s=|S|=2, \ldots, n
$$

and we define

$$
B[s]:=\sum_{|S|=s} B_{S} \text { and } B:=\sum_{s=2}^{n} B[s]
$$

Theorem 2.3 The moduli spaces $\overline{\mathcal{M}}_{0, n}\left(\mathbb{P}^{1}, 1\right)$ and $\overline{\mathcal{M}}_{0, n}$ are smooth projective algebraic varieties. Moreover, the boundary is a divisor with strict normal crossings.

Remark 2.4 In particular, the pair $\left(\overline{\mathcal{M}}_{0, n}\left(\mathbb{P}^{1}, 1\right), B\right)$ is log-canonical. 


\subsection{Resolution for the full moduli space}

We obtain a good resolution $(\tilde{X}, \tilde{D})$ of $(X, D)$ using the above formalism. Consider the quotient map

$$
q: \overline{\mathcal{M}}_{0, n}\left(\mathbb{P}^{1}, 1\right) \rightarrow \tilde{X}:=\overline{\mathcal{M}}_{0, n}\left(\mathbb{P}^{1}, 1\right) / \mathfrak{S}_{n}
$$

Let $\tilde{D}[s]$ and $\tilde{D}$ be the images of $B[s]$ and $B$ under this map.

Proposition 2.5 The map $q$ is ramified only along the boundary B. At the generic points of $B[2]$ the ramification has order 2 . For all $s=3, . ., n$, the map $q$ is unramified at the generic points of $B[s]$. We have the formula

$$
q^{*}\left(K_{\tilde{X}}+\tilde{D}\right)=K_{\overline{\mathcal{M}}_{0, n}\left(\mathbb{P}^{1}, 1\right)}+B
$$

and $(\tilde{X}, \tilde{D})$ is log-canonical.

Proof. The map $q$ ramifies at points corresponding to stable maps

$$
\left(C, p_{1}, \ldots, p_{n}, \mu\right)
$$

that admit an automorphism permuting the marked points. The ramification order is the order of this automorphism group. If the images of the $n$ points under $\mu$ are distinct then there is no automorphism of $\mu$ permuting them. This proves the first assertion. If marked points coincide there is an irreducible component $\mathbb{P}^{1} \subset C$ which is collapsed by $\mu$ and which contains these points. If there are two such points this component admits an automorphism of order two exchanging the points and fixing the point of intersection with the rest of $C$. This proves the second assertion. If there are more than two marked points then there is generally no such automorphism. This proves the third assertion.

The ramification formula and the fact that the pair $(\tilde{X}, \tilde{D})$ is log-canonical follow from an easy local computation combined with Remark 2.4 (see Propositions 20.2 and 20.3 of [17]).

Take $\mathfrak{S}_{n}$-quotients of the point map to obtain a birational map

$$
\varrho: \tilde{X} \rightarrow \mathbb{P}\left(\operatorname{Sym}^{n} V^{*}\right),
$$

assigning to $p_{1}, \ldots, p_{n} \in \mathbb{P}^{1}$ a polynomial vanishing at these points. The boundary divisor $\tilde{D}[2]$ is the proper transform of the discriminant $D$ under $\varrho$. The boundary divisors $\tilde{D}[s]$ (for $s \geq 3$ ) are the exceptional divisors for $\varrho$. 


\subsection{Resolution of the generic orbit}

Let $\alpha:=\left(\alpha_{1}, \ldots, \alpha_{n}\right)$ be a set of distinct complex numbers and $f=f_{\alpha}$ the binary form of degree $n$ with roots $\alpha_{j}$. Let $C_{\alpha} \in \mathcal{M}_{0, n}$ be the corresponding pointed rational curve and $\mu_{\alpha} \in \mathcal{M}_{0, n}\left(\mathbb{P}^{1}\right)$ the corresponding map. The fiber

$$
Y_{\alpha}:=\psi^{-1}\left(C_{\alpha}\right) \subset \overline{\mathcal{M}}_{0, n}\left(\mathbb{P}^{1}, 1\right)
$$

contains $\mu_{\alpha}$. Let $\tilde{X}_{f}$ be the image of $Y_{\alpha}$ under the quotient map $q$ and $\tilde{D}_{f}$ its intersection with the boundary $\tilde{D}$. This coincides with the general fiber of the map

$$
\psi^{\prime}: \tilde{X}=\overline{\mathcal{M}}_{0, n}\left(\mathbb{P}^{1}, 1\right) \rightarrow \overline{\mathcal{M}}_{0, n} / \mathfrak{S}_{n} .
$$

The map $\varrho$ induces a resolution

$$
\varrho_{f}: \tilde{X}_{f} \rightarrow X_{f}
$$

with $\varrho_{f}\left(\tilde{D}_{f}\right)=D_{f}$.

To describe the $Y_{\alpha}$ explicitly, we use the tower

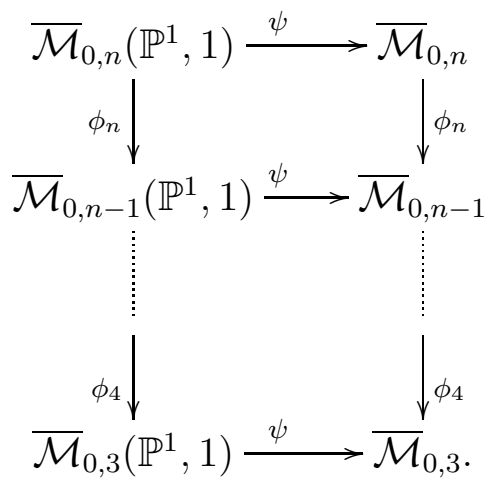

When $n=3, \overline{\mathcal{M}}_{0,3}=$ point and $Y_{\alpha} \simeq \overline{\mathcal{M}}_{0,3}\left(\mathbb{P}^{1}, 1\right)$, which is isomorphic to the product $\left(\mathbb{P}^{1}\right)^{3}$ blown up along the small diagonal $\Delta_{\text {small }}$. The boundary divisors correspond to the following stable maps

$$
B[2]=\frac{-\mathrm{j}}{\mathrm{1}_{\mathrm{k}}}, B[3]=\underset{ }{\text { f }} .
$$

In the above pictures the collapsed components are represented by vertical lines. Note that the normal bundle

$$
\mathcal{N}_{\Delta} \cong \mathcal{O}(2) \oplus \mathcal{O}(2)
$$


so that the exceptional divisor $E=B[3] \simeq \mathbb{P}^{1} \times \mathbb{P}^{1}$. Let

$$
\pi_{1}: E \rightarrow \mathbb{P}^{1}
$$

be the projection to the cross ratio of the marked points and the node and

$$
\pi_{2}: E \rightarrow \mathbb{P}^{1}
$$

the projection onto the image of the collapsed curve.

The divisor $B[2]$ is the proper transform of $\Delta$, the large diagonal.

For the arbitrary degree case, we analyze the failure of the block squares in the tower to be fiber products. Given a generic

$$
C_{\alpha}=\left(\mathbb{P}^{1}, \alpha_{1}, \ldots, \alpha_{n}\right) \in \mathcal{M}_{0, n}, \quad \alpha_{i} \neq \alpha_{j},
$$

we compare the fibers

$$
Y_{\alpha_{1}, \ldots, \alpha_{n}}=\psi^{-1}\left(C_{\alpha}\right) \text { and } Y_{\alpha_{1}, \ldots, \alpha_{n-1}}=\psi^{-1}\left(\phi_{n}\left(C_{\alpha}\right)\right)=\psi^{-1}\left(\mathbb{P}^{1}, \alpha_{1}, \ldots, \alpha_{n-1}\right)
$$

using the forgetting map

$$
\phi_{n}: \overline{\mathcal{M}}_{0, n}\left(\mathbb{P}^{1}, 1\right) \rightarrow \overline{\mathcal{M}}_{0, n-1}\left(\mathbb{P}^{1}, 1\right) .
$$

Given a stable map

$$
\left(C, \alpha_{1}, \ldots, \alpha_{n}, \mu\right) \in \psi^{-1}\left(C_{\alpha}\right)
$$

there are three cases to consider:

1. $C=\mathbb{P}^{1}$;

2. $C=\mathbb{P}^{1} \cup \mathbb{P}^{1}$ with the collapsed component containing $\alpha_{1}, \ldots, \alpha_{n}$;

3. $C=\mathbb{P}^{1} \cup \mathbb{P}^{1}$ with the collapsed component containing $\alpha_{1}, \ldots, \alpha_{n-1}$ but not $\alpha_{n}$.

Case 1:

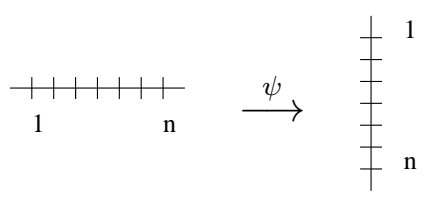


Case 2:

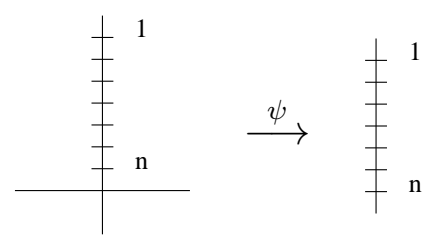

Case 3:

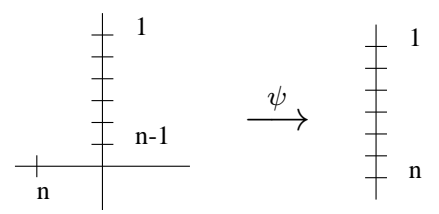

Over the open subset of $Y_{\alpha_{1}, \ldots, \alpha_{n}}$ corresponding to the first two cases, $\phi_{n}$ induces an isomorphism between $Y_{\alpha_{1}, \ldots, \alpha_{n}}$ and $Y_{\alpha_{1}, \ldots, \alpha_{n-1}}$. In the third case, we forget the image of the $n$-th marked point. The map $\phi_{n}$ blows up the locus in $Y_{\alpha_{1}, \ldots, \alpha_{n-1}}$ where $\alpha_{1}, \ldots, \alpha_{n-1}$ are on the collapsed component and $\alpha_{n}$ coincides with the node (of attachment). This is a curve isomorphic to $\mathbb{P}^{1} \subset\left(B[n-1] \cap Y_{\alpha_{1}, \ldots, \alpha_{n-1}}\right)$ : The generic map takes the form:

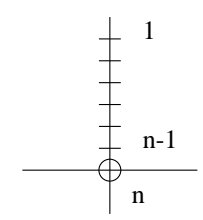

We summarize the above discussion in the following

Proposition 2.6 Let $\alpha_{1}, \ldots \alpha_{n}$ be distinct complex numbers. The forgetting maps induce a sequence of birational morphisms

$$
Y_{\alpha_{1}, \ldots, \alpha_{n}} \stackrel{\phi_{n}}{\longrightarrow} Y_{\alpha_{1}, \ldots, \alpha_{n-1}} \ldots \stackrel{\phi_{4}}{\longrightarrow} Y_{\alpha_{1}, \alpha_{2}, \alpha_{3}} \simeq \overline{\mathcal{M}}_{0,3}\left(\mathbb{P}^{1}, 1\right) .
$$

The moduli space of stable maps $\overline{\mathcal{M}}_{0,3}\left(\mathbb{P}^{1}, 1\right)$ is isomorphic to $\left(\mathbb{P}^{1}\right)^{3}$ blown up along the small diagonal with exceptional divisor $E \simeq \mathbb{P}^{1} \times \mathbb{P}^{1}$. The map $\phi_{j}$ blows up the proper transform of $\pi_{1}^{-1}\left(\alpha_{j}\right)$. In particular, $Y_{\alpha_{1}, \ldots, \alpha_{n}}$ is smooth and its boundary has strict normal crossings, contained in $B[n-1] \cup B[n]$.

Remark 2.7 We are blowing up along disjoint curves, so the order of the blow-up does not matter. 
Proposition 2.8 Let $f$ be a generic binary form of degree $n \geq 3$ with roots $\alpha_{1}, \ldots, \alpha_{n}$. Then the restriction of $q$ to $X_{f}$ is ramified only along the boundary $B \cap X_{f}$. At generic points of $(B[n] \cup B[n-1]) \cap Y_{\alpha}$, the restriction of $q$ is unramified. We have the formula

$$
q^{*}\left(K_{\tilde{X}_{f}}+\tilde{D}_{f}\right)=K_{Y_{\alpha}}+\left[Y_{\alpha} \cap B\right]
$$

and $\left(\tilde{X}_{f}, \tilde{D}_{f}\right)$ is log-canonical.

Proof. The argument is similar to the one in Proposition 2.5, and is omitted.

\section{Verification of exponents}

\subsection{Explicit basis of $\operatorname{Pic}\left(Y_{\alpha}\right)$}

Write

$$
\operatorname{Pic}\left(\left(\mathbb{P}^{1}\right)^{3}\right)=\mathbb{Z} g_{1}+\mathbb{Z} g_{2}+\mathbb{Z} g_{3}, \quad g_{i}=\operatorname{pr}_{i}^{*}\left(c_{1}\left(\mathcal{O}_{\mathbb{P}^{1}}(+1)\right)\right),
$$

with large diagonals

$$
\Delta_{i j}=g_{i}+g_{j}-E, B[2]=2\left(g_{1}+g_{2}+g_{3}\right)-3 E .
$$

By Proposition 2.6, $Y_{\alpha}$ is obtained by blowing up the $(n-3)$ sections of

$$
\pi_{2}: B[3] \rightarrow \mathbb{P}^{1}
$$

Let $F_{4}, \ldots, F_{n}$ denote the corresponding exceptional divisors and identify $E$ and its proper transform. Relabel

$$
\begin{aligned}
F_{k} & =\Delta_{i j},\{i, j, k\}=[1,2,3] \\
& =g_{i}+g_{j}-E-F_{4}-\cdots-F_{n},
\end{aligned}
$$

so that $\mathfrak{S}_{n}$ acts on the $F_{k}, k=1, \ldots, n$, in the obvious way. Note that $E$ and the $F_{k}$ generate $\operatorname{Pic}\left(Y_{\alpha}\right)$.

Proposition 3.1 The $\mathfrak{S}_{n}$-stable boundary divisors

$$
\begin{aligned}
A[n-1] & =F_{1}+\cdots+F_{n} \\
A[n] & =E
\end{aligned}
$$


generate the $\mathfrak{S}_{n}$-invariant Picard group of $Y_{\alpha}$, and $A[j]=B[j] \cap Y_{\alpha}$. The canonical class of $Y_{\alpha}$ is

$$
\begin{aligned}
K & =-2\left(g_{1}+g_{2}+g_{3}\right)+E+2\left(F_{4}+\cdots+F_{n}\right) \\
& =-A[n-1]-2 A[n]
\end{aligned}
$$

\subsection{Computation of the effective cone}

Lemma 3.2 The $\mathfrak{S}_{n}$-invariant effective cone of $Y_{\alpha}$ is generated by the classes $A[n]$ and $A[n-1]$.

Proof. We apply the method of $\$ 1.3$. The class $A[n]$ is exceptional and thus a generator of the effective cone. To show that $A[n-1]$ is the second generator, we exhibit a nef curve not intersecting $A[n-1]$. Consider the $\mathbb{G}_{m}$-action on $\mathbb{P}^{1}$ :

$$
\rho_{t}:(z, w) \mapsto(t z, w) .
$$

We may assume that the points $\alpha_{1}, \ldots, \alpha_{n}$ are not contained in the fixed point locus of $\rho_{t}$. Any singular element in the orbit closure is:

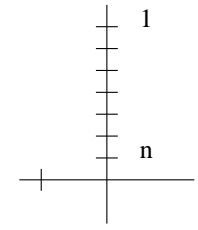

where the point of attachment is $0($ or $\infty$ ) and the other labelled point is $\infty$ (resp. 0). This is disjoint from $A[n-1]$.

\subsection{Proof of Theorem 2.1}

Proof. The Kodaira energy for $\left(X_{f}, D_{f}\right)$ can be computed on $\left(\tilde{X}_{f}, \tilde{D}_{f}\right)$, by Propositions 1.3 and 2.8. By Propositions 1.4 and 2.6, it suffices to compute the Kodaira energy for $\left(Y_{\alpha}, A[n]+A[n-1]\right)$. Recall there is a composed morphism

$$
\beta: Y_{\alpha} \stackrel{q}{\rightarrow} X_{f} \hookrightarrow \mathbb{P}\left(\mathrm{Sym}^{n} V^{*}\right) \simeq \mathbb{P}^{n}
$$

Lemma 3.3 The pull-back of the hyperplane class takes the form

$$
L=:\left[\beta^{*} \mathcal{O}_{\mathbb{P}^{n}}(+1)\right]=\frac{1}{2}((n-2) A[n-1]+n A[n]) .
$$


Proof. Let $R$ be the class of a curve in $A[n]$ corresponding to

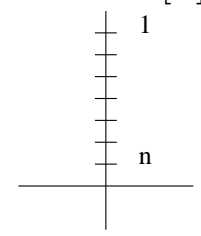

with varying point of attachment on the collapsed component. This is the proper transform of the generic fiber of the map $\pi_{1}: E \rightarrow \mathbb{P}^{1}$. Then

$$
\begin{aligned}
&\left.A[n-1]\right|_{R}=n=\#\left\{\begin{array}{l}
\frac{1, \ldots, \mathrm{j}-1, \mathrm{j}+1, \ldots, \mathrm{n}}{|+|} \mid-\mathrm{j} \\
\left.A[n]\right|_{R}
\end{array}=1, \ldots, n\right\} \\
&-1-(n-3)=2-n .
\end{aligned}
$$

For the second intersection number, note that $A[n]=E$ and apply the blowup description of Proposition 2.6. In $\overline{\mathcal{M}}_{0,3}\left(\mathbb{P}^{1}, 1\right)$ we have

$$
E=\mathbb{P}\left(\mathcal{N}_{\Delta_{\text {small }}}\right)=\mathbb{P}^{1} \times \mathbb{P}^{1}
$$

and $\mathcal{N}_{E}=\mathcal{O}(-1)$. After blowing up $(n-3)$ further sections of

$$
E \rightarrow \Delta_{\text {small }}
$$

the normal bundle is reduced to $\mathcal{O}(-1-(n-3))$.

We know that $A[n]=B[n] \cap Y_{\alpha}$ is collapsed by the map $\beta$, so

$$
\beta^{*} \mathcal{O}(1)=c((n-2) A[n-1]+n A[n])
$$

for some $c \in \mathbb{N}$. Since

$$
\begin{aligned}
(n-2) A[n-1]+n A[n] \\
\quad=2\left((n-2)\left(g_{1}+g_{2}+g_{3}\right)-(n-3) E-(n-2)\left(F_{4}+\cdots+F_{n}\right)\right) .
\end{aligned}
$$

the claim follows.

We have

$$
\begin{aligned}
K_{Y_{\alpha}}+\left[Y_{\alpha} \cap B\right] & =K_{Y_{\alpha}}+A[n]+A[n-1]=-A[n] \\
K_{Y_{\alpha}}+\left[Y_{\alpha} \cap B\right]+\alpha L & =\alpha \frac{n-2}{2} A[n-1]+\left(\alpha \frac{n}{2}-1\right) A[n],
\end{aligned}
$$


and by definition

$$
a\left(\beta^{*} L\right):=\inf \left\{\alpha \mid \alpha \beta^{*} L+K_{Y_{\alpha}}+\left[Y_{\alpha} \cap B\right] \in \Lambda_{\mathrm{eff}}\left(Y_{\alpha}\right)\right\}
$$

Hence Lemma 3.2 yields

$$
a\left(L,\left[Y_{\alpha} \cap B\right]\right)=2 / n .
$$

Thus $a\left(L, D_{f}\right)=2 / n$, as desired!

\section{The $\mathfrak{S}_{n}$-invariant effective cone of the full moduli space}

In this section, we compute the $\mathfrak{S}_{n}$-invariant part of the effective cone of $\overline{\mathcal{M}}_{0, n}\left(\mathbb{P}^{1}, 1\right)$, its canonical class, and the Kodaira energy of the line bundle $L:=\beta^{*} \mathcal{O}_{\mathbb{P}^{n}}(+1)$, where

$$
\beta=\rho \circ q: \overline{\mathcal{M}}_{0, n}\left(\mathbb{P}^{1}, 1\right) \stackrel{q}{\rightarrow} \tilde{X} \stackrel{\rho}{\rightarrow} \mathbb{P}^{n}
$$

We will also compute the Kodaira energy of $H:=\rho^{*} \mathcal{O}_{\mathbb{P}^{n}}(+1)$.

We first recall some basic facts about $\overline{\mathcal{M}}_{0, n}\left(\mathbb{P}^{1}, 1\right) \simeq \mathbb{P}^{1}[n]$, following [11]. In addition to the divisor classes $B_{S}$ introduced above, we shall also consider

$$
L_{a}:=\left\{\left(C, p_{1}, \ldots, p_{n}, \mu\right) \in \overline{\mathcal{M}}_{0, n}\left(\mathbb{P}^{1}, 1\right): \mu\left(p_{a}\right)=0 \in \mathbb{P}^{1}\right\}, \quad a=1, \ldots, n .
$$

The cohomology $H^{*}\left(\overline{\mathcal{M}}_{0, n}\left(\mathbb{P}^{1}, 1\right)\right)$ is generated by the classes $L_{a}$ and $B_{S}$, subject to the relations

1. $L_{a}^{2}=0$;

2. $B_{S} \cdot B_{S^{\prime}}=0$ for $S \cap S^{\prime} \neq \emptyset$;

3. $\left(L_{a}-L_{a^{\prime}}\right) B_{S}=0$ for $a, a^{\prime} \in S$;

4. $\left(\sum_{S \supset\left\{a, a^{\prime}\right\}} B_{S}\right)=L_{a}+L_{a^{\prime}}$, for $1 \leq a<a^{\prime} \leq n$. 
The generators of the $\mathfrak{S}_{n}$-invariant subspace are

$$
L:=\sum_{a=1}^{n} L_{a}, \quad B[s]=\sum_{|S|=s} B_{S}, \quad 2 \leq s \leq n .
$$

After averaging over $\mathfrak{S}_{n}$

$$
(n-1) L=\sum_{s=2}^{n} \frac{s(s-1)}{2} B[s] .
$$

Theorem 4.1 The classes $D[2], \ldots, D[n]$ generate the effective cone of $Y$. The classes $B[2], \ldots, B[n]$ generate the $\mathfrak{S}_{n}$-invariant effective cone of the moduli space $\overline{\mathcal{M}}_{0, n}\left(\mathbb{P}^{1}, 1\right)$.

Proof. We implement the strategy of $\$ 1.3$ with

$$
\Gamma=\{B[2], \ldots, B[n]\}
$$

This entails finding curve classes that are nef relative to $\Gamma$. Let

$$
M=\sum_{j=2}^{n} d_{j} B[j]
$$

denote an $\mathfrak{S}_{n}$-invariant divisor class with no boundary divisors as fixed components.

Recall the description of the boundary divisor $B_{S}$ :

$$
\begin{aligned}
B_{S} & \simeq \overline{\mathcal{M}}_{0, n+1-s}\left(\mathbb{P}^{1}, 1\right) \times \overline{\mathcal{M}}_{0, s+1}, & & s=|S|>2, \\
& \simeq \overline{\mathcal{M}}_{0, n-1}\left(\mathbb{P}^{1}, 1\right), & & s=2 .
\end{aligned}
$$

Take $s \geq 3$ and let $C_{s} \subset B_{S}$ be the class of the generic fiber of the map

$$
\overline{\mathcal{M}}_{0, n+1-s}\left(\mathbb{P}^{1}, 1\right) \times \overline{\mathcal{M}}_{0, s+1} \rightarrow \overline{\mathcal{M}}_{0, n+1-s}\left(\mathbb{P}^{1}, 1\right) \times \overline{\mathcal{M}}_{0, s}
$$

forgetting the attaching point. Since $C_{s}$ passes through the generic point of $B_{S}$, averaging $C_{s}$ over $\mathfrak{S}_{n}$ yields a curve class which is nef relative to $\Gamma$. In particular, for each $\mathfrak{S}_{n}$-invariant divisor $M=\sum_{j=2}^{n} d_{j} B[j]$, moving relative to $\Gamma$, we have $C_{s} \cdot M \geq 0$. 
We compute intersections of $C_{s}$ with the various elements of $\Gamma$. First, the map $\beta$ blows down the divisors $B_{S}$ for $|S| \neq 2$; the data of the collapsed component is lost completely. It follows that $L \cdot C_{s}=0$. A simple combinatorial analysis gives

$$
C_{s} \cdot B_{T}= \begin{cases}1 & \text { if } T=S-\{\sigma\} \\ 0 & \text { otherwise, unless } T=S .\end{cases}
$$

which means that $C_{s} \cdot B[s-1]=s$. Relation 1 gives

$$
0=B_{S} \cdot C_{s} \frac{s(s-1)}{2}+s \frac{(s-1)(s-2)}{2}
$$

so $B_{S} \cdot C_{s}=-(s-2)$. To summarize, we have

$$
C_{s} \cdot B[j]=\left\{\begin{array}{cl}
s & \text { if } j=s-1 \\
-(s-2) & \text { if } j=s \\
0 & \text { otherwise }
\end{array}\right.
$$

Using this information, we extract inequalities on the coefficients of $M$. The condition $M \cdot C_{s} \geq 0$ yields

$$
s d_{s-1} \geq(s-2) d_{s}
$$

so we get a chain of inequalities:

$$
d_{n} \leq \frac{n}{n-2} d_{n-1} \leq \frac{n(n-1)}{(n-2)(n-3)} d_{n-2} \leq \ldots \leq \frac{n(n-1)}{2} d_{2}
$$

If some $d_{s}<0$ then $d_{j}<0$ for each $j \geq s$.

We consider another curve class in $B_{S}$ to get inequalities in the reverse direction. Fix $s \geq 2$ and let $R_{s}$ denote the class of the generic fiber of

$$
\overline{\mathcal{M}}_{0, n+1-s}\left(\mathbb{P}^{1}, 1\right) \times \overline{\mathcal{M}}_{0, s+1} \rightarrow \overline{\mathcal{M}}_{0, n-s}\left(\mathbb{P}^{1}, 1\right) \times \overline{\mathcal{M}}_{0, s+1}
$$

induced by forgetting $\tau$, one of the $n+1-s$ points not contained in $S$. Again, $R_{s}$ passes through the generic point of $B_{S}$, so averaging over $\mathfrak{S}_{n}$ yields a curve class such that $R_{s} \cdot M \geq 0$. 
We compute intersections as before. The map $\beta$ sends $R_{s}$ to a line in $\mathbb{P}^{n}$, i.e., the linear forms with $n-1$ fixed roots and one varying root. It follows that $L \cdot R_{s}=1$. The line $R_{s}$ intersects $B_{T}$ properly in the following cases

$$
R_{s} \cdot B_{T}= \begin{cases}1 & \text { if } T=S \cup\{\tau\} ; \\ 1 & \text { if } T=\{\tau, v\}, v \notin S ; \\ 0 & \text { otherwise, unless } T=S .\end{cases}
$$

Summing over $\mathfrak{S}_{n}$-orbits gives

$$
R_{s} \cdot B[j]=\left\{\begin{array}{cl}
1 & \text { if } j=s+1 \\
n-s-1 & \text { if } j=2 ; \\
0 & \text { otherwise, unless } j=s .
\end{array}\right.
$$

Applying Relation [, we find

$$
(n-1)=s(s-1) / 2 R_{s} \cdot B[s]+(s+1) s / 2+(n-s-1),
$$

so $R_{S} \cdot B_{S}=R_{s} \cdot B[s]=-1$.

We extract the inequalities

$$
d_{s+1}-d_{s} \geq(n-s-1) d_{2} ;
$$

in particular, if $d_{2}>0$ then each $d_{j}>0$. Adding together the inequalities

$$
\begin{aligned}
d_{n}-d_{n-1} & \geq 0 \\
d_{n-1}-d_{n-2} & \geq d_{2} \\
& \cdots \\
d_{4}-d_{3} & \geq(n-4) d_{2} \\
d_{3} & \geq(n-2) d_{2} .
\end{aligned}
$$

gives

$$
d_{n} \geq \frac{n^{2}-5 n+8}{2} d_{2} .
$$

Combining with inequality 2, we obtain

$$
\left(n^{2}-1\right) d_{2} \geq\left(n^{2}-5 n+8\right) d_{2},
$$

hence $d_{2}>0$. 
Theorem 4.2 On the moduli space of stable maps $\left(\overline{\mathcal{M}}_{0, n}\left(\mathbb{P}^{1}, 1\right), B\right)$ and its $\mathfrak{S}_{n}$-quotient $(Y, D)$, we have

$$
a(B, L)=a(D, H)=2 / n \text {. }
$$

Proof. We proceed to calculate the canonical class:

$$
K_{\overline{\mathcal{M}}_{0, n}\left(\mathbb{P}^{1}, 1\right)}=-2 L+\sum_{s=3}^{n} B[s](s-2)
$$

This follows from the explicit blowup realization of the Fulton-MacPherson configuration space $\mathbb{P}^{1}[n]=\overline{\mathcal{M}}_{0, n}\left(\mathbb{P}^{1}, 1\right)\left[11\right.$. The exceptional divisors $B_{S}$ (for $s=|S| \geq 3$ ) arise from blowing up centers in codimension $s-1$.

We compute the log-Kodaira energy of $L$ on $\overline{\mathcal{M}}_{0, n}\left(\mathbb{P}^{1}, 1\right)$ with respect to the boundary $B$. We have

$$
\begin{aligned}
K+B+a L & =(a-2) L+\sum_{s=2}^{n}(s-1) B[s] \\
& =(a-2)\left(\sum_{s=2}^{n} \frac{s(s-1)}{2(n-1)} B[s]\right)+\sum_{s=2}^{n}(s-1) B[s] \\
& =\sum_{s=2}^{n}(s-1)\left(\frac{s(a-2)}{2(n-1)}+1\right) B[s]
\end{aligned}
$$

which is effective if and only if

$$
a \geq \frac{2(s-n+1)}{s}, \quad s=2, \ldots, n \text {. }
$$

The most restrictive inequality occurs when $s=n$, where we obtain $a \geq 2 / n$. Consequently, $a(L, B)=2 / n$, as expected.

The Kodaira energy for $(X, D)$ can be computed on $(\tilde{X}, \tilde{D})$, by Propositions 1.3 and 2.5. By Proposition 1.4 and Theorem [2.3, it is equal to the Kodaira energy for $\left(\overline{\mathcal{M}}_{0, n}\left(\mathbb{P}^{1}, 1\right), B\right)$. 


\section{Final remarks}

A) The orbit closure $X_{f}$ depends on the form $[f]$. We get equivariant compactifications of $\mathrm{PGL}_{2}$ depending on moduli. This dependence is made abundantly clear in the blow-up description of Proposition 2.6.

B) The pair $\left(\overline{\mathcal{M}}_{0, n}, \delta\right)$ is of log general type: $K_{\overline{\mathcal{M}}_{0, n}}+\delta$ is ample and log canonical (see, for example, $\S 7.1$ of [13]). The map

$$
\psi: \overline{\mathcal{M}}_{0, n}\left(\mathbb{P}^{1}, 1\right) \rightarrow \overline{\mathcal{M}}_{0, n}
$$

is a log-Fano fibration onto a log-variety of general type (or the point, when $n=3)$.

C) The pair $\left(\overline{\mathcal{M}}_{0, n}, \delta\right)$ satisfies Vojta's conjecture. We realize $\mathcal{M}_{0, n}$ as an open subset of an algebraic torus with explicit complement. Fix $n-1$ points in $\mathbb{P}^{n-3}$ in general position. Consider the set $\mathcal{H}$ of $\frac{1}{2}(n-1)(n-2)$ hyperplanes spanned by $n-3$ of the fixed points. Kapranov [15] has shown that $\mathcal{M}_{0, n} \simeq$ $\mathbb{P}^{n-3}-\cup_{H \in \mathcal{H}} H$. The torus is obtained by excising the $n-2$ hyperplanes spanned by subsets of the first $n-2$ of the points.

D) We therefore expect that the asymptotic behavior of integral points of $\overline{\mathcal{M}}_{0, n}\left(\mathbb{P}^{1}, 1\right)$ with respect to the boundary is obtained by summing the contributions of the integral points on the fibers of $\psi$. This explains why the Kodaira energies for the moduli space of stable maps (Theorem 4.2) and the fibers of $\psi$ (Theorem 2.1) should coincide (for the case of rational points, see [2]).

\section{References}

[1] V. Batyrev and Yu. I. Manin, Sur le nombre des points rationnels de hauteur bornée des variétés algebriques, Math. Ann. 286 (1990), 27-43.

[2] V. Batyrev and Yu. Tschinkel, Tamagawa numbers of polarized algebraic varieties, Astérisque 251 (1998), 299-340.

[3] B. J. Birch, Forms in many variables, Proc. Roy. Soc. Ser. A 265 (1961/1962), 245-263. 
[4] A. Chambert-Loir and Yu. Tschinkel, On the distribution of points of bounded height on equivariant compactifications of vector groups, Invent. Math. 148 (2002), no. 2, 421-452.

[5] M.C. Chang and Z. Ran, The Kodaira dimension of the moduli space of curves of genus 15, J. Differential Geom. 24 (1986), no. 2, 205-220.

[6] W. Duke, Z. Rudnick, and P. Sarnak, Density of integer points on affine homogeneous varieties, Duke Math. J. 71 (1993), no. 1, 143-179.

[7] A. Eskin and C. McMullen, Mixing, counting, and equidistribution in Lie groups, Duke Math. J. 71 (1993), no. 1, 181-20.

[8] A. Eskin, S. Mozes, and N. Shah, Unipotent flows and counting lattice points on homogeneous varieties, Ann. of Math. (2) 143 (1996), no. 2, 253-299.

[9] J. Franke, Yu. Manin, and Yu. Tschinkel, Rational points of bounded height on Fano varieties, Invent. Math. 95 (1989), 421-435.

[10] T. Fujita, Kodaira energies of polarized log varieties, Journ. Math. Soc. Japan 48 (1996), no. 1, 1-12.

[11] W. Fulton and R. MacPherson, A compactification of configuration spaces, Ann. of Math. (2) 139 (1994), no. 1, 183-225.

[12] W. Fulton and R. Pandharipande, Notes on stable maps and quantum cohomology, Algebraic geometry - Santa Cruz 1995, 45-96, Proc. Sympos. Pure Math., 62, Part 2, Amer. Math. Soc., Providence, RI, 1997.

[13] B. Hassett, Moduli spaces of weighted pointed stable curves, to appear in Advances in Mathematics.

[14] B. Hassett and Yu. Tschinkel, On the effective cone of the moduli space of pointed rational curves, Topology and Geometry: Commemorating SISTAG, A.J. Berrick, M.C. Leung, and X.W. Xu eds., Contemporary mathematics 314, 83-96, American Mathematical Society, Providence, 2002 .

[15] M. Kapranov, Veronese curves and Grothendieck-Knudsen moduli space $\bar{M}_{0, n}$, J. Algebraic Geom. 2 (1993), no. 2, 239-262. 
[16] S. Keel and J. McKernan, Contraction of extremal rays on $\overline{\mathcal{M}}_{0, n}$, alg-geom 9607009.

[17] J. Kollar, Flips and abundance for algebraic threefolds, Astérisque 211, (1992).

[18] F. Knudsen, The projectivity of the moduli space of stable curves. II. The stacks $M_{g, n}$, Math. Scand. 52 (1983), no. 2, 161-199.

[19] M. Kontsevich, Enumeration of rational curves via torus actions, The moduli space of curves (Texel Island, 1994), 335-368, Progr. Math., 129, Birkhäuser, Boston, 1995.

[20] M. Kontsevich and Yu. Manin, Gromov-Witten classes, quantum cohomology, and enumerative geometry, Comm. Math. Phys. 164 (1994), no. $3,525-562$.

[21] E. Peyre, Hauteurs et nombres de Tamagawa sur les variétés de Fano, Duke Math. J. 79 (1995), 101-218.

[22] E. Peyre, Terme principal de la fonction zeta des hauteurs et torseurs universels, Astérisque 251 (1998), 259-298.

[23] E. Peyre and Yu. Tschinkel, Tamagawa numbers of diagonal cubic surfaces of higher rank, alg-geom 0009092, Rational points on algebraic varieties, Progr. Math. 199, Birkhäuser, Boston, 2001.

[24] W. Rulla, The Birational Geometry of $\overline{\mathcal{M}}_{3}$ and $\overline{\mathcal{M}}_{2,1}$, Ph.D. Thesis, University of Texas at Austin (2001).

[25] W. Schmidt, The density of integer points on homogeneous varieties, Acta Math. 154 no. 3-4, (1985), 243-296.

[26] J. Silverman, Integral points on curves and surfaces, Number theory (Ulm, 1987), 202-241, Lecture Notes in Mathematics 1380, Springer, New York, 1989.

[27] P. Vojta, Diophantine approximations and value distribution theory, Lecture Notes in Mathematics 1239, Springer, Berlin, 1987. 
Brendan Hassett

Department of Mathematics

Rice University, MS 136

6100 S. Main Street

Houston, TX 77005-1892, U.S.A.

hassett@math.rice.edu

Yuri Tschinkel

Department of Mathematics

Princeton University

Fine Hall, Washington Road

Princeton, NJ 08544-1000, U.S.A.

ytschink@math.princeton.edu 